\title{
How to exploit sparsity in RNNs on event-driven architectures
}

\author{
Jarno Brils \\ Eindhoven University of Technology \\ Eindhoven, The Netherlands \\ jarno.brils@gmail.com
}

\author{
Luc Waeijen \\ GrAI Matter Labs \\ Eindhoven, The Netherlands \\ lwaeijen@graimatterlabs.ai
}

\author{
Arash Pourtaherian \\ GrAI Matter Labs \\ Eindhoven, The Netherlands \\ apourtaherian@graimatterlabs.ai
}

\begin{abstract}
Event-driven architectures have been shown to provide low-power, low-latency artificial neural network (ANN) inference. This is especially beneficial on Edge devices, particularly when combined with sparse execution. Recurrent neural networks (RNNs) are ANNs that emulate memory. Their recurrent connection enables the reuse of previous output for the generation of new output. However, when trying to use RNNs in a sparse context on event-driven architectures, novel challenges in synchronization and the usage of sparse data are encountered. In this work, these challenges are systematically analyzed, and mechanisms to overcome them are proposed. Experimental results of a monocular depth estimation use case on the NeuronFlow architecture show that sparsity in RNNs can be exploited effectively on event-driven architectures.
\end{abstract}

\section{CCS CONCEPTS}

- Hardware $\rightarrow$ Asynchronous circuits; • Computer systems organization $\rightarrow$ Embedded hardware; Embedded software; Neural networks.

\section{KEYWORDS}

sparsity, neural networks, monocular depth estimation, recurrent neural networks, asynchronous, event-driven

\section{ACM Reference Format:}

Jarno Brils, Luc Waeijen, and Arash Pourtaherian. 2021. How to exploit sparsity in RNNs on event-driven architectures. In 24th International Workshop on Software and Compilers for Embedded Systems (SCOPES '21), November 1-2, 2021, Eindhoven, Netherlands. ACM, New York, NY, USA, 6 pages. https://doi.org/10.1145/3493229.3493302

\section{INTRODUCTION}

In recent years, asynchronous edge computing has risen in number of uses and in usability. By shifting (pre)processing of sensor data close to the sensors, large reductions in data transfer and centralized compute requirements are made. Naturally, not all tasks are feasible to be run on the edge. For example, edge devices may not process data fast enough, or data processing might draw more power than what is available. One of the tasks that are increasingly often performed on edge devices is Artificial Neural Network (ANN) inference. Such ANNs can be used for face recognition or object

Permission to make digital or hard copies of all or part of this work for personal or classroom use is granted without fee provided that copies are not made or distributed for profit or commercial advantage and that copies bear this notice and the full citation on the first page. Copyrights for components of this work owned by others than the author(s) must be honored. Abstracting with credit is permitted. To copy otherwise, or republish, to post on servers or to redistribute to lists, requires prior specific permission and/or a fee. Request permissions from permissions@acm.org.

SCOPES '21, November 1-2, 2021, Eindhoven, Netherlands

() 2021 Copyright held by the owner/author(s). Publication rights licensed to ACM. ACM ISBN 978-1-4503-9166-5/21/06 ..\$15.00

https://doi.org/10.1145/3493229.3493302 detection in surveillance cameras, or monocular depth estimation in drones. To run ANN inference in energy and compute constrained edge devices, limitations to the complexity of networks must be taken into account. A way to reduce the number of computations is the exploitation of sparsity. In case the compute architecture supports dynamic scheduling of instructions, or asynchronous execution, sparsity enables skipping superfluous operations.

One of the types of ANNs that have had limited use on edge devices due to inherent complexities is Recurrent Neural Networks (RNNs). These RNNs use temporal relations between inputs to emulate memory in ANNs. This enables a form of memory in otherwise stateless neural networks. Especially when processing real-time input on edge devices, RNNs can result in noticeable improvements in inference results. However, using RNNs in a sparse execution environment poses serious challenges, especially when executed on event-driven architectures. This work systematically analyzes the challenges of using RNNs on asynchronous architectures, and describes the mechanisms which are imperative to enable their evaluation on such machines. Results are evaluated on the industrial use case of monocular depth estimation using an ANN with a Long Short-Term Memory (LSTM) layer on the NeuronFlow architecture.

The major contributions of this work can be summarized as follows:

- An approach that enables deployment of existing RNNs on asynchronous machines without retraining.

- Evaluation of the proposed approach on the industrial usecase of monocular depth estimation using an LSTM layer.

The remainder of this works is structured as follows. In section 2 , related work on asynchronous architectures, and sparse ANNs and RNNs is outlined. Section 3 analyzes the challenges posed by RNN inference on these types of architectures, and proposes solutions to these challenges. Results based on both experimental and analytical evaluation are described in section 4 .

\section{RELATED WORK}

Many chip architectures have tried to imitate the brain. Such machines are typically referred to as neuromorphic architectures [12]. Neuromorphic engineering can be based on conventional digital transistor logic, memristors, or can even be fully analog. What most neuromorphic chips have in common is their usage of a spiking neuron model. This means that, as with the brain, neurons fire asynchronously. Combined with a high degree of parallelism, many calculations can be performed in a short time.

In recent years several businesses in the semiconductor industry have released their version of a neuromorphic chip. In 2014 IBM introduced TrueNorth [13], which has a capacity of one million spiking neurons, and consumes well below $100 \mathrm{~mW}$ on real-time video input. Intel launched the many-core Loihi chip, which has the potential to scale up massively, in 2018 [2]. Every core contains an 
engine that enables learning on-chip. Before Loihi, the SpiNNaker Project [4] used a very large array of custom chips to evaluate spiking neural networks. GrAI Matter Lab's NeuronFlow [14] combines a spiking neuron model and a dataflow design methodology in the design of GML's first chip: GrAIOne [15]. Their architecture allows for low latency and low power execution of dataflow graphs and spiking neural networks using a many-core grid of neuron cores that communicate using events.

Sparse execution of neural networks benefits from propagating changes in values (deltas) through the network instead of full values. Sigma-delta encoding with discretized deltas has been shown to result in a significant reduction in operation count [18]. Other work has expanded upon this by enabling conversion of regular, pre-trained neural networks to spiking neural networks [7, 21]. The addition of thresholding logic to suppress propagation of small deltas reduces computation counts even further [11]. Sparse execution of neural networks is far more efficient on asynchronous architectures [22], than on statically scheduled architectures such as CPUs and GPUs.

Existing research in sparse execution of RNNs has focused solely on theoretical applications or architectures supporting only RNN layers. For example, Neil et al. [17] show how sparsity and delta inputs can be used for gated recurrent units (GRUs). They do not mention sparse outputs of the GRU and do not explore the costs of running this tail sparsely. Based on these formulas, Gao et al. proposed two delta RNN accelerators, DeltaRNN (2018) [5] and EdgeDRNN (2020) [6]. Both are very efficient executors of sparse GRU layers, and start by encoding non-sparse input to sparse vectors. These sparse vectors are then used in the RNN gates, after which the tail operations are executed non-sparsely. Both of these architectures only support GRU layers, and let other network layers be handled by a separate CPU. Gupta et al. introduce a modular accelerator for sparse RNNs in 2019 [8]. This accelerator is more flexible than both delta RNN accelerators, and supports both simple RNNs and GRUs. However, as with the other architectures, the focus is on executing single RNN layers sparsely by encoding non-sparse inputs. LSTMs are not supported, and output is non-sparse.

In this work, more generalized implementations are proposed. Although LSTMs are the focus, simple RNNs and GRUs can be implemented using the same methodology. Furthermore, the challenges of asynchronous execution of an RNN layer in a larger network are tackled. Contrary to existing work, the proposed implementations can be used in existing sparse, asynchronous ANN execution architectures, without, or with only minor, architecture modifications.

\section{METHODS}

This section explains the challenges and proposed solutions for the implementation of RNNs on existing asynchronous, sparse architectures. First, to better understand the limitations and details of RNNs, their workings are further elaborated in subsection 3.1 . Then, implementation challenges are outlined in subsection 3.2, followed by proposed solutions to these challenges in subsection 3.3.

\subsection{Background}

Recurrent neural networks differ from conventional ANNs by their recurrent connection [3]. Output $y_{t}$ at timestep $t$ depends on both input $x_{t}$ and the previous timestep's output $y_{t-1}$. Several variants of RNNs exist. The most basic variant (simple $R N N$ ) multiplies the recurrent inputs with weights, and accumulates this with the weighted inputs, as expressed in Equation 1. $W$ and $U$ are input and recurrent weights, respectively, $b$ is a bias value, and $\sigma$ denotes an activation function (typically hyperbolic tangent or sigmoid).

$$
y_{t}=\sigma\left(W \cdot x_{t}+U \cdot y_{t-1}+b\right)
$$

When training the weights for RNNs, the backpropagation through time (BPTT) algorithm is commonly used [19]. The RNN layer is unrolled for every element in the training sequence, backpropagating through the same layer multiple times. This drastically increases the probability of gradients vanishing or exploding. Alternative RNNs (hereafter referred to as complex RNNs) alleviate this problem.

One such complex RNN is Long Short-Term Memory (LSTM) [9]. An LSTM cell consists of four RNN gates, that each perform the operations that the simple RNN performs (with differing weights and activation functions). The resulting values of these RNN gates are used to update the internal cell state and calculate the output value. In this work, the group of operations that happen after the RNN gates will be referred to as the LSTM tail. The equations for an LSTM cell are listed in Equation 2.

$$
\begin{aligned}
f_{t} & =\sigma_{g}\left(W_{f} \cdot x_{t}+U_{f} \cdot y_{t-1}+b_{f}\right) \\
i_{t} & =\sigma_{g}\left(W_{i} \cdot x_{t}+U_{i} \cdot y_{t-1}+b_{i}\right) \\
o_{t} & =\sigma_{g}\left(W_{o} \cdot x_{t}+U_{o} \cdot y_{t-1}+b_{o}\right) \\
\tilde{c}_{t} & =\sigma_{h}\left(W_{c} \cdot x_{t}+U_{c} \cdot y_{t-1}+b_{c}\right) \\
c_{t} & =f_{t} \circ c_{t-1}+i_{t} \circ \tilde{c}_{t} \\
y_{t} & =o_{t} \circ \sigma_{h}\left(c_{t}\right)
\end{aligned}
$$

Another complex RNN is the gated recurrent unit (GRU) [1], which is similar to the LSTM, but uses fewer weights and computations. It does however have a dependency between the output of one of its RNN gates and the input of another RNN gate. In this work, the focus lies on LSTMs, though all challenges and solutions also apply to other RNN variants with minor modifications.

\subsection{Challenges of sparse, asynchronous inference of RNNs}

There are two main challenges that are encountered when attempting to use RNNs on sparse and asynchronous architectures: the need for synchronization, and the usage of delta values for sparsity.

The pollution problem is a major issue that is encountered when attempting to run RNNs asynchronously. Since output $y_{t}$ at timestep $t$ of an RNN is dependent on both input $x_{t}$ and recurrent input $y_{t-1}$, we must ensure that no other data arrives before all necessary data has arrived. In fully asynchronous execution, an input belonging to $x_{t+1}$ may arrive before all previous recurrent inputs $y_{t-1}$ have arrived. When this happens, pollution of the frame occurs, as output $y_{t}$ is based on inputs not corresponding to time $t$. If this happens frequently, computed outputs may significantly drift from the expected outputs. Pollution can be considered an anti-dependency problem, where writing output must happen after reading all inputs.

Furthermore, if an event's consumer is also the event's producer, more problems may occur. Suppose that arriving events immediately get processed, independently of whether all outputs have been 
generated. In this case, a recurrent input $y_{t}^{\prime}$ belonging to timestep $t$ may get multiplied and accumulated into the neuron state before all outputs $y_{t} \in Y$ have been generated. When this happens, the next output $y_{t}^{\prime \prime}$ is no longer based on the correct state, but uses a polluted state, resulting in incorrect output. This self-pollution problem is similar to the pollution problem for general asynchronous RNN execution. If there is only a single computation unit (like a core) that is responsible for a neuron and the generation of its output, deadlocking may occur instead. If the network cannot accommodate all produced output events of a neuron, outputs need to be consumed before more can be produced. However, the computation unit that is responsible for consuming this event is still busy producing events. Therefore, the event blocks, and backpressure causes event generation to block as well, resulting in a deadlock.

Making complex RNNs work with sparse input and output is more complex than making regular ANNs sparse. A common characteristic of all types of RNNs is the RNN gate. LSTMs use four of these, GRUs use three, and the simple RNN consists of a single RNN gate. Inside this gate, inputs and recurrent inputs are weighted, accumulated, and the activated results are then output. For simple RNNs, this output is then immediately used as the recurrent input for the next timestep, whereas for the complex RNNs such as LSTMs and GRUs, more operations follow using the results of the gates.

Regular ANN layers such as fully connected or convolution layers can easily be made sparse. All that is required is a state storage for every neuron. The (delta) output $y_{t}$ of a sparse ANN neuron at timestep $t$ is defined as $\Delta y_{t}=\sigma\left(s_{t}\right)-\sigma\left(s_{t-1}\right)$, where the state is defined as $s_{t}=W \cdot \Delta x_{t}+s_{t-1}$, and $\sigma$ is the neuron's activation function. $\Delta x_{t}$ is the sparse input at timestep $t$, and $W$ contains the neuron weights. The RNN gate can be made sparse using the same principle. The only difference between an RNN gate and a fully connected or convolution layer is that it has two weight matrices, one for inputs, and one for recurrent inputs. However, by concatenating the weight matrices, the RNN gate can be transformed to a regular ANN layer, and can use sparsity in the same way.

The main complexity with sparsity and RNNs is therefore not in the RNN gate, but in the operations that follow afterwards in complex RNNs such as LSTMs and GRUs. This tail uses the RNN gates' results to perform some multiplications, additions, subtractions, or activations. For LSTMs, the tail consists of the operations in the last two lines of Equation 2, where $f_{t}, i_{t}, \tilde{c}_{t}$, and $o_{t}$ are RNN gate outputs at time $t, \sigma_{h}$ is an activation function, $c_{t-1}$ is the previous cell state, $\circ$ indicates elementwise multiplications, and + denotes elementwise addition. In this case (and for GRUs as well), elementwise multiplication is the most occurring operation (in any case, addition can be done sparsely without changes). Suppose we have a multiplication $a=b \cdot c$ of two operands $b$ and $c$, both of which can be sparse. The result at timestep $t$ would then be: $a_{t}=\left(\Delta b+b_{t-1}\right) \cdot\left(\Delta c+c_{t-1}\right)=\Delta b \cdot \Delta c+b_{t-1} \cdot \Delta c+\Delta b \cdot c_{t-1}+b_{t-1} \cdot c_{t-1}$. That means that to get sparse or delta output of $a$, we would need to do: $\Delta a=a_{t}-a_{t-1}=a_{t}-b_{t-1} \cdot c_{t-1}=\Delta b \cdot \Delta c+b_{t-1} \cdot \Delta c+\Delta b \cdot c_{t-1}$. This means that storing just the previous state $a_{t-1}=b_{t-1} \cdot c_{t-1}$ is not enough, as states for both operands are needed to multiply with the sparse inputs. This means that in any implementation, state storage doubles, and more memory interactions and multiply-accumulate (MAC) operations are required. This significantly increases the compute cost, and would only be beneficial if sparsity is very high.

\subsection{Proposed solutions}

The challenges that have been outlined can all be overcome. First of all, the pollution problem can be solved by using a synchronization mechanism when processing input. The use for synchronization is two-fold in this case. Firstly, synchronization is used to make output event generation wait until all inputs and recurrent inputs have arrived. Secondly, this makes sure that new input generation is blocked until existing input has been processed. Synchronization can be implemented by sending synchronization signals or events, waiting for two of these to arrive (one signaling the end of input, one signaling the end of recurrent input), and then triggering output generation. This does assume that events that have the same source and destination will not overtake each other (so end-of-frame signals do not get processed before all frame inputs have been processed).

As for the prevention of self-pollution and potential deadlocking, the problem is only solvable by introducing an extra buffer step between event consumption and recurrent event production. For every output, this buffer only needs to store a single value. As long as consumption of recurrent events can then be guaranteed (by splitting consumption and production over two computation units, or by increasing event consumption priority), deadlocking or selfpollution will no longer happen. Luckily, for complex RNNs such as LSTMs and GRUs, there are additional computations to be done after recurrent event consumption, so the buffer stage is already built-in (assuming that the entire complex LSTM or GRU cell is not implemented as a single neuron).

In RNNs, the expectation is that sparsity in the tail is limited, due to recurrent inputs continually updating the outputs, even if inputs do not change. Even then, only a very small portion of the operations in these complex RNNs are done in the tail. For example, for the LSTM implementation that is tested in this work, this is only 250 thousand of the nearly 20 million worst-case total operations. Therefore, the better solution would be to perform all operations in the tail of the LSTM on full values, and not on sparse delta values. To still benefit from sparsity in the output and the recurrent input, the outputs of the RNN can be converted back to sparse delta values by comparing the output to the previous timestep's output. This also allows for thresholding to be implemented, to further increase sparsity at the expense of accuracy.

\section{EXPERIMENTS AND RESULTS}

The proposed solutions for using RNNs on sparse and asynchronous architectures are tested on a monocular depth estimation application. Monocular depth estimation produces a depth map from a 2D image input. Depth estimation can be used for object avoidance in drones or in pick-and-place robots in industrial automation. The network that is used is based on FastDepth [20], a state of the art monocular depth estimation network optimized for embedded systems. The FastDepth network has an encoder-decoder structure and is based on MobileNet [10]. Since the purpose of this work is to enable the usage of RNNs on edge devices, a relatively small network is chosen and enhanced with an LSTM layer. This layer is added after the base FastDepth network. It uses a convolutional LSTM with a filter size of $7 \times 7$, and sigmoid activation. Event-based simulation is done using a custom dataflow simulator, that can be 
setup to process events and data like a generic asynchronous architecture. This custom dataflow simulator gives detailed metrics down to event levels, indicating event and firing activity, and allowing for deduction of the number of executed MAC instructions.

\subsection{Implementation on NeuronFlow}

For evaluation the NeuronFlow architecture is selected because it is specialized in asynchronous sparse ANN inference on the Edge. Its many-core grid is used to execute ANN neuron operations. These neurons multiply inputs with stored weights, accumulate into a neuron state, and perform activation on the output. The neuron supports sparse inputs and / or outputs. To increase sparsity, a threshold value can be set. Synchronization is done by processing batches of data at a time (for example: one frame at a time), halting input of the next batch until all inputs have been processed.

The RNN gates of the LSTM layer are mapped to conventional ANN neurons that are setup to receive delta inputs, and output full values. These full values go through the tail of the LSTM, after which the output is converted into a sparse delta value again. The elementwise operations in the tail are mapped to the functionality of ANN neurons. For elementwise multiplications, one of two input operands is repurposed as the weight of the neuron. When using linear activation, this implementation is equivalent to ordinary elementwise multiplication. The addition in the LSTM tail is done by multiplying the input by an identity weight of one, and accumulating it into the neuron state. This does require delta values as inputs to the addition, since the neuron state is not cleared between time steps. Luckily, the inputs for the addition in the LSTM tail both originate in ANN neurons that perform multiplication. By having these ANN neurons produce delta output, the input condition for the add neuron is met. Since activation can also be done in an ANN neuron, the activation function from the LSTM tail is implemented in the add neuron. Note that the state value of the add neuron (the cell state) is used as one of the inputs to the multiplication. Since the activation function is only performed on outputs of the neuron, the state value remains the unactivated cell state, as is desired. Finally, the multiply neuron that produces the output values is set to output sparse delta values. This means that inputs and outputs of the LSTM layer are all deltas. This implementation is visualized in Figure 1. Dashed arrows indicate state values that are repurposed as weights, and solid arrows indicate regular input events.

\subsection{Results}

For each of the established challenges, results are reported, along with overall details.

It is difficult to show results for the synchronization implementation. Using a simple simulation, a demonstration is given instead that shows both the need for synchronization, and the validity of the solution. In Figure 2, the results of an experiment that simulates a simple RNN with four outputs are visualized. Asynchronous simulation results are compared to reference results from the same network in TensorFlow. For the first four frames / inputs, recurrent inputs and normal inputs are provided in order, followed by an output trigger signal. For these frames, output of the simulation is identical to the expected results. For frame 4, inputs for frame 5 are fed alongside inputs for the current frame. This means that once

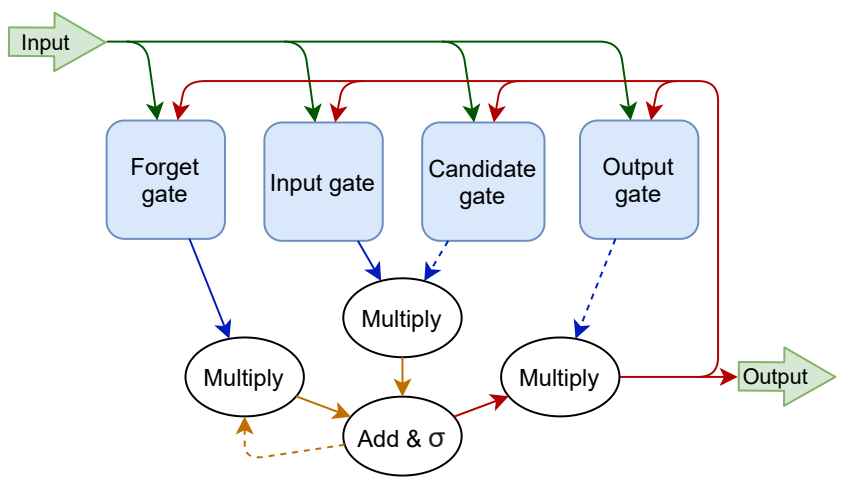

Figure 1: Visualization of the mapping of an LSTM cell to ANN neurons. Filled arrows indicate events, dashed arrows indicate state reads.

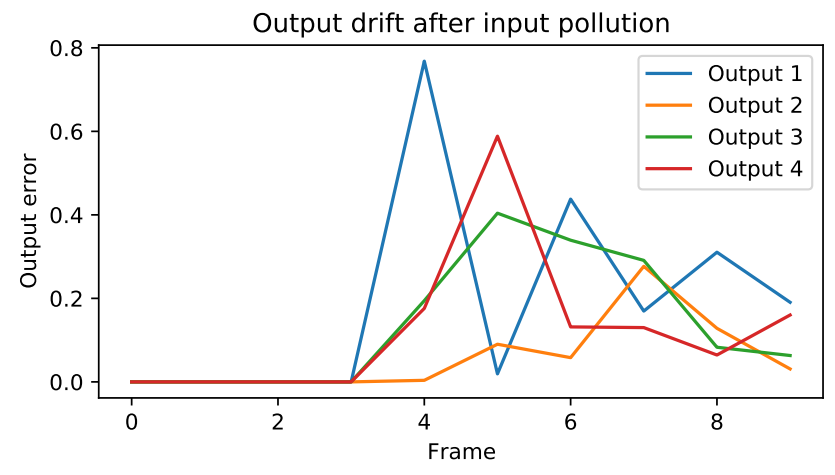

Figure 2: Visualization of drift from the expected results after pollution is introduced in frame 4 .

the output is generated, it is based on more inputs than expected. This clearly results in incorrect outputs. In all later frames, inputs again arrive in the predefined, correct order. However, once the value on the recurrent input of the RNN has been incorrect, it never recovers. Output will stay incorrect. This shows that if the trigger signal that is sent after the inputs of the first four frames is also used for synchronization, correct output is guaranteed. This synchronization signal follows all expected inputs, and future inputs are blocked until the signal has been processed.

The other synchronization issue, self-pollution or deadlocking for single-step cycles, is more difficult to show. Since event-generation in the created simulator is done for all output events in a single step, the problem cannot be simulated using this simulator. This is because the problem only occurs when production of output events is still ongoing when an input event is received. Instead, the problem and solutions are proven using a detailed analysis. Suppose a core is responsible for processing all $n$ neurons of a simple RNN layer, and suppose that the maximum capacity of the event buffers is $k<n$ events. The execution unit / core has just finishing processing all inputs for the first neuron in the layer, and has started sending recurrent events. These events are sent to all $n$ neurons in the RNN layer. After $k$ events are sent, event buffers are full. Now, an event 
in the event buffer must be consumed to prevent deadlocking. One of two things can happen now, depending on the architecture:

- The execution unit is busy producing events, and cannot consume the event. Event production deadlocks, and so does the execution unit.

- The event is consumed, and the internal state of the receiving neuron is updated using the event value. If this neuron has not yet produced all of its output events, the output events that are next sent from this neuron are based on an updated state. This self-pollution results in incorrect output.

To solve these issues, a buffer state is introduced. When a neuron is updated, a single event is sent to the buffer, instead of sending all $n$ recurrent events. Once all buffers have been updated, the buffers will then send the recurrent events. In this case, event consumption must still be guaranteed. If the buffers belong to a different execution unit than the neurons, events can immediately be consumed. If the buffers belong to the same execution unit, deadlocking may still occur. Therefore, recurrent input event must always be consumable, even when event production is still ongoing. This can be done by increasing recurrent input event priority on NeuronFlow. Since the old state has been buffered, self-pollution can no longer occur.

The reasoning for the limitations to sparsity in RNNs has been explained in subsection 3.3. The necessity for multiple states and thereby more operations and memory accesses for sparse execution of the tail operations of complex RNNs has been mathematically proven. For simple RNNs, this is not needed. The initial frames in Figure 2 were executed on sparse inputs, and show no difference from expected outputs. To evaluate what it would cost to execute the tail of an LSTM layer sparsely, a numerical analysis is done.

The tail of an LSTM consists of three multiplication operations, one activation function call, and an addition of two values. Multiplication of two sparse values for sparse output $(\Delta a=\Delta b \cdot \Delta c+$ $\left.b_{t-1} \cdot \Delta c+\Delta b \cdot c_{t-1}\right)$ requires state storage for both inputs, and performs three multiplications and two additions (three MAC operations). It also performs two state memory reads and writes. Addition of two sparse values does not need any special consideration $\left(\Delta a=a_{t}-a_{t-1}=\left(\left(\Delta b+b_{t-1}\right)+\left(\Delta c+c_{t-1}\right)\right)-\left(b_{t-1}+c_{t-1}\right)=\Delta b+\Delta c\right)$. Activation of sparse input does require storage of the old state, since the non-linear nature of activation functions requires full state input. Overall, this means that for the sparse execution of one output in the tail of an LSTM, $3 \cdot 3+2 \cdot 1+1 \cdot 1=12$ MAC operations and $3 \cdot 2+2 \cdot 0+1 \cdot 1=7$ memory reads and writes are required Non-sparse execution in the suggested implementation requires $3 \cdot 1+2 \cdot 1+1 \cdot 1=6$ MAC operations, and $3 \cdot 1+2 \cdot 1=5$ memory reads and $2 \cdot 1=2$ memory writes. Note that these numbers do not include architecture-specific operations and memory interactions, such as event or weight retrieval. This means that even if we assume instant and no-cost memory interactions, 50\% sparsity would be the minimum before sparse execution becomes worth the extra effort. However, it is highly likely that memory interactions are orders of magnitude more costly (in terms of latency and in terms of energy usage) than MAC operations. Therefore, sparsity requirements would likely need to be significantly higher before sparse execution is worth it. Since these sparsity numbers are very unlikely to be reached, full-state tail operations in complex RNNs are strongly advised.

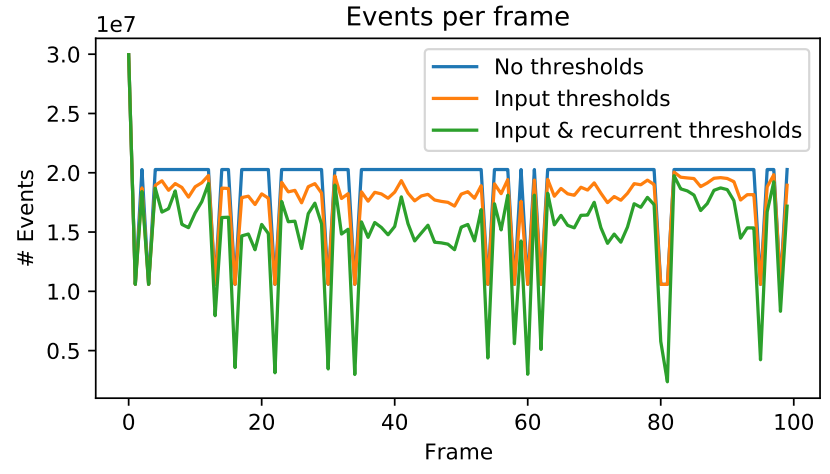

Figure 3: Processed events per input frame all input types.

To get proper results on the effects of sparsity on the computational load of LSTM inference, simulations are performed for three different input types. A SpArNet simulation of the FastDepth network is performed to get outputs for sparse inference of the base network. The input data for the base network simulation are from the NYU depth v2 dataset [16], namely the first 100 frames of the 'kitchen_0056a' sequence. The outputs of this base network are fed into the LSTM layer. The three input types are as follows:

- Sparse output of the base network without thresholds

- Sparse output of the base network with thresholds targeting $50 \%$ sparsity of the inputs

- Sparse output of the base network with thresholds targeting $50 \%$ sparsity of the inputs and recurrent inputs

Before simulating, some static worst-case metrics can be calculated. For the given implementation, the LSTM's RNN gates account for $224^{2} \cdot 7^{2} \cdot 4 \cdot 2=19,668,992$ consumed events. The operations in the tail require a further $224^{2} \cdot(5+2)=351,232$ events. This results in a worst-case total of 20,020,224 consumed events per frame for the LSTM, without sparsity. The worst-case number of produced events, assuming synchronized output, is much lower, at only 401,408. This shows that the majority of events are processed at the LSTM layer's input. High input sparsity would therefore have large effects on the total number of operations.

An overview of the number of simulated events per frame in the input sequence is shown in Figure 3. The most interesting features in this figure are the irregular low spikes in event counts. These occur when the input data for two consecutive frames are identical, and unchanging input is skipped. Even though recurrent input to the LSTM does change if input does not, it seems that new input influences the output (sparsity) more than recurrent input. Furthermore, a clear difference between the different input data types is seen. Even though events in the tail of the LSTM are not sparse, sparse input reduces event and computation load.

In Figure 4, the effects of sparsity on output accuracy can be seen. As expected, not using thresholds results in identical results to those of a reference simulation in TensorFlow. However, if input and / or recurrent input to the LSTM is sparse, some difference can be expected. Remarkably, increasing thresholds for the recurrent input seems to cause little extra difference. This may signify that either the output is not very dependent on the recurrent input, or 


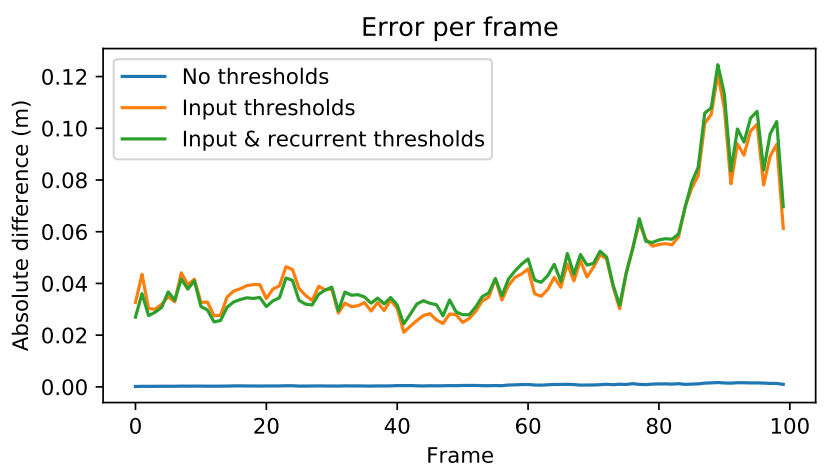

Figure 4: Error per input frame for all input types.

recurrent input thresholds can be further raised without problems. Note that even in the worst case, the absolute difference to the reference output was only twelve centimeters on a reference range of ten meters for depth estimation on the NYU depth v2 dataset. Furthermore, this is absolute difference to reference simulation results, and not to ground truth, so higher thresholds might even decrease the estimation error with respect to the ground truth.

\section{CONCLUSION}

This work has shown that using RNNs sparsely on generic sparse asynchronous architectures is feasible. Especially architectures that are tailored to sparse neural network inference require few changes to work with RNNs. One must however take care that some synchronization is performed to prevent state pollution, and possibly even deadlocking. This is due to the defining characteristic of RNNs: the cyclic dependency of output and input. We have shown that the monocular depth estimation task can be done with low latency on sparse, asynchronous low-power edge devices, even when extended with RNN functionality. By exploiting sparsity, even in RNNs, latency can be reduced further. In the future, an investigation into the modification of sparse neural network computer architectures for RNN inference may prove beneficial. If performance of RNN layer inference can be further improved, overall latency may drop further. Furthermore, an in-depth investigation into the trade-off between sparsity and accuracy was not the focus of this work, but may provide insights into how (and how much) to further reduce inference costs for sparse, asynchronous RNNs.

\section{ACKNOWLEDGMENTS}

Prof. Dr. Henk Corporaal for guidance and assistance during the research process proceeding this work.

\section{REFERENCES}

[1] Kyunghyun Cho, Bart van Merrienboer, Çaglar Gülçehre, Fethi Bougares, Holger Schwenk, and Yoshua Bengio. 2014. Learning Phrase Representations using RNN Encoder-Decoder for Statistical Machine Translation. CoRR abs/1406.1078 (2014). http://arxiv.org/abs/1406.1078

[2] M. Davies, N. Srinivasa, T. Lin, G. Chinya, Y. Cao, S. H. Choday, G. Dimou, P. Joshi, N. Imam, S. Jain, Y. Liao, C. Lin, A. Lines, R. Liu, D. Mathaikutty, S. McCoy, A. Paul, J. Tse, G. Venkataramanan, Y. Weng, A. Wild, Y. Yang, and H. Wang. 2018 Loihi: A Neuromorphic Manycore Processor with On-Chip Learning. IEEE Micro 38,1 (2018), 82-99.
[3] Jeffrey L. Elman. 1990. Finding structure in time. Cognitive Science 14, 2 (1990), 179-211. https://doi.org/10.1016/0364-0213(90)90002-E

[4] Steve Furber and Steve Temple. 2007. Neural Systems Engineering. Journal of the Royal Society, Interface / the Royal Society 4 (05 2007), 193-206. https: //doi.org/10.1098/rsif.2006.0177

[5] Chang Gao, Daniel Neil, Enea Ceolini, Shih-Chii Liu, and Tobi Delbruck. 2018. DeltaRNN: A Power-Efficient Recurrent Neural Network Accelerator. In Proceedings of the 2018 ACM/SIGDA International Symposium on Field-Programmable Gate Arrays (Monterey, CALIFORNIA, USA) (FPGA '18). Association for Computing Machinery, New York, NY, USA, 21-30. https://doi.org/10.1145/3174243.3174261

[6] C. Gao, A. Rios-Navarro, X. Chen, S. C. Liu, and T. Delbruck. 2020. EdgeDRNN: Recurrent Neural Network Accelerator for Edge Inference. IEEE fournal on Emerging and Selected Topics in Circuits and Systems 10, 4 (2020), 419-432. https: //doi.org/10.1109/JETCAS.2020.3040300

[7] S. Ghosh-Dastidar and H. Adeli. 2009. Third Generation Neural Networks: Spiking Neural Networks. In Advances in Computational Intelligence, Wen Yu and Edgar N. Sanchez (Eds.). Springer Berlin Heidelberg, Berlin, Heidelberg, 167-178.

[8] Udit Gupta, Brandon Reagen, Lillian Pentecost, Marco Donato, Thierry Tambe, Alexander M. Rush, Gu-Yeon Wei, and David Brooks. 2019. MASR: A Modular Accelerator for Sparse RNNs. arXiv:1908.08976 [eess.SP]

[9] S. Hochreiter and J. Schmidhuber. 1997. Long Short-Term Memory. Neural Computation 9, 8 (1997), 1735-1780. https://doi.org/10.1162/neco.1997.9.8.1735

[10] Andrew G. Howard, Menglong Zhu, Bo Chen, Dmitry Kalenichenko, Weijun Wang, Tobias Weyand, Marco Andreetto, and Hartwig Adam. 2017. MobileNets: Efficient Convolutional Neural Networks for Mobile Vision Applications. CoRR abs/1704.04861 (2017). http://arxiv.org/abs/1704.04861

[11] M. A. Khoei, A. Yousefzadeh, A. Pourtaherian, O. Moreira, and J. Tapson. 2020. SpArNet: Sparse Asynchronous Neural Network execution for energy efficient inference. In 2020 2nd IEEE International Conference on Artificial Intelligence Circuits and Systems (AICAS). 256-260. https://doi.org/10.1109/AICAS48895. 2020.9073827

[12] C. Mead. 1990. Neuromorphic electronic systems. Proc. IEEE 78, 10 (1990), 1629-1636. https://doi.org/10.1109/5.58356

[13] Paul A. Merolla, John V. Arthur, Rodrigo Alvarez-Icaza, Andrew S. Cassidy, Jun Sawada, Filipp Akopyan, Bryan L. Jackson, Nabil Imam, Chen Guo, Yutaka Nakamura, Bernard Brezzo, Ivan Vo, Steven K. Esser, Rathinakumar Appuswamy, Brian Taba, Arnon Amir, Myron D. Flickner, William P. Risk, Rajit Manohar, and Dharmendra S. Modha. 2014. A million spiking-neuron integrated circuit with a scalable communication network and interface. Science 345, 6197 (2014), 668-673. https://doi.org/10.1126/science. 1254642

[14] O. Moreira, A. Yousefzadeh, F. Chersi, G. Cinserin, R.-J. Zwartenkot, A. Kapoor, P. Qiao, P. Kievits, M. Khoei, L. Rouillard, A. Ferouge, J. Tapson, and A. Visweswara. 2020. NeuronFlow: a neuromorphic processor architecture for Live AI applications. In Conference on Design, Automation and Test in Europe.

[15] O. Moreira, A. Yousefzadeh, F. Chersi, A. Kapoor, R. . Zwartenkot, P. Qiao, G. Cinserin, M. A. Khoei, M. Lindwer, and J. Tapson. 2020. NeuronFlow: A Hybrid Neuromorphic - Dataflow Processor Architecture for AI Workloads. In 2020 2nd IEEE International Conference on Artificial Intelligence Circuits and Systems (AICAS). 01-05.

[16] Pushmeet Kohli Nathan Silberman, Derek Hoiem and Rob Fergus. 2012. Indoor Segmentation and Support Inference from RGBD Images. In ECCV.

[17] D. Neil, J. H. Lee, T. Delbruck, and S. Liu. 2017. Delta Networks for Optimized Recurrent Network Computation. In Proceedings of the 34th International Conference on Machine Learning (Proceedings of Machine Learning Research, Vol. 70), Doina Precup and Yee Whye Teh (Eds.). PMLR, International Convention Centre, Sydney, Australia, 2584-2593. http://proceedings.mlr.press/v70/neil17a.html

[18] Peter O'Connor and Max Welling. 2017. Sigma Delta Quantized Networks. In 5th International Conference on Learning Representations, ICLR 2017, Toulon, France, April 24-26, 2017, Conference Track Proceedings. OpenReview.net. https: //openreview.net/forum?id=HkNRsU5ge

[19] A. J. Robinson and Frank Fallside. 1987. The Utility Driven Dynamic Error Propagation Network. Technical Report CUED/F-INFENG/TR.1. Engineering Department, Cambridge University, Cambridge, UK

[20] Diana Wofk, Fangchang Ma, Tien-Ju Yang, Sertac Karaman, and Vivienne Sze. 2019. FastDepth: Fast Monocular Depth Estimation on Embedded Systems. CoRR abs/1903.03273 (2019). http://arxiv.org/abs/1903.03273

[21] A. Yousefzadeh, S. Hosseini, P. Holanda, S. Leroux, T. Werner, T. SerranoGotarredona, B. L. Barranco, B. Dhoedt, and P. Simoens. 2019. Conversion of Synchronous Artificial Neural Network to Asynchronous Spiking Neural Network using sigma-delta quantization. In 2019 IEEE International Conference on Artificial Intelligence Circuits and Systems (AICAS). 81-85. https: //doi.org/10.1109/AICAS.2019.8771624

[22] A. Yousefzadeh, M. A. Khoei, S. Hosseini, P. Holanda, S. Leroux, O. Moreira, J. Tapson, B. Dhoedt, P. Simoens, T. Serrano-Gotarredona, and B. Linares-Barranco. 2019. Asynchronous Spiking Neurons, the Natural Key to Exploit Temporal Sparsity. IEEE Journal on Emerging and Selected Topics in Circuits and Systems 9, 4 (2019), 668-678. https://doi.org/10.1109/JETCAS.2019.2951121 